\title{
Philosophiques
}

\section{L’équilibre de la raison et de la croyance}

\section{Richard Vaillancourt}

Volume 43, numéro 2, automne 2016

URI : https://id.erudit.org/iderudit/1038216ar

DOI : https://doi.org/10.7202/1038216ar

Aller au sommaire du numéro

Éditeur(s)

Société de philosophie du Québec

ISSN

0316-2923 (imprimé)

1492-1391 (numérique)

Découvrir la revue

Citer ce document

Vaillancourt, R. (2016). L'équilibre de la raison et de la croyance. Philosophiques, 43(2), 471-480. https://doi.org/10.7202/1038216ar d'utilisation que vous pouvez consulter en ligne.

https://apropos.erudit.org/fr/usagers/politique-dutilisation/ 


\title{
L'équilibre de la raison et de la croyance
}

\author{
RICHARD VAILLANCOURT \\ Cégep Bois-de-Boulogne \\ richard.vaillancourt@bdeb.qc.ca
}

Qu'on soit d'accord ou non avec la position de Georges Leroux en ce qui concerne le cours Éthique et culture religieuse ${ }^{1}$, ceux qui aiment la philosophie devraient se réjouir qu'un d'entre eux puisse jouer un rôle important dans l'élaboration de politiques publiques au Québec. Nous pouvons être heureux qu'un grand spécialiste de la philosophie grecque participe à l'orientation de la société québécoise dans un des domaines où elle en a le plus besoin, en éducation. Il est aussi rare qu'une politique concrète soit défendue avec une telle transparence et soumise à une discussion politique aussi féconde, exception faite de quelques procès d'intentions ou autres sophismes que le débat a malheureusement vu naître. On constate avec la publication de Différence et liberté: enjeux actuels de l'éducation au pluralisme que, par son souci de clarté et de justification rationnelle, la philosophie permet d'assainir le débat démocratique; l'État québécois tirerait un grand profit à dialoguer en permanence avec les philosophes québécois et, dans le cas de l'éducation au pluralisme, Georges Leroux représente ceux-ci dignement.

Le Québec a récemment effectué un important virage en s'engageant dans l'éducation au pluralisme moral et religieux. Leroux pense les fondements de ce virage à partir de la reconnaissance de la différence dans une perspective humaniste. À première vue, cela pourrait sembler paradoxal: l'humanisme ne cherche-t-il pas à penser l'être humain au-delà de ses différences sociales particulières? Ne pose-t-il pas comme essence la liberté et l'autonomie de l'être humain par rapport à l'autorité de la tradition? Mais l'humanisme est aussi étroitement lié à la tolérance et au respect de la différence, ce qui suscite une question fondamentale: "Comment conjuguer, au sein de la diversité sociale, respect, tolérance et recherche rationnelle de principes communs ? ${ }^{2} »(\mathrm{p} .36)$.

Leroux l'admet d'emblée, il est impossible pour l'État d'adopter la neutralité axiologique, position qui serait même non souhaitable: "Un projet humaniste ne peut que [la] refuser, car cette position conduit à l'appauvrissement spirituel et moral de tous»(p. 30I). L'État a une mission éthique et politique de transmission, de formation et de construction d'une culture publique commune qui évolue au sein d'un dialogue social. Ce qu'il

1. Que j'identifierai maintenant par l'acronyme ECR.

2. Pour éviter la multiplication des notes de bas de page, je mets le numéro de page entre parenthèses. Sauf indication contraire, les citations proviennent de Différence et liberté: enjeux actuels de l'éducation au pluralisme. 
propose dans la foulée du rapport Parent est une véritable philosophie publique de l'éducation qui s'engage dans l'éducation à la démocratie par une définition commune "des valeurs universelles de citoyenneté " (p. II9). S'agit-il pour autant d'un républicanisme rigide à la française, intolérant à la différence? Leroux défend une perspective humaniste qui s'apparente au républicanisme quant à l'importance d'une culture commune dépassant le cadre des droits individuels, et quant à la valorisation de l'idéal d'autonomie et de liberté. Mais là où le républicanisme traditionnel vise une unicité parfois intolérante, Leroux propose une ouverture plus substantielle à la différence et au pluralisme. L'humanisme qu'il propose s'abreuve à des sources multiples, contrairement à ce que nous propose le modèle français. En ce sens, il se situe aussi dans le sillon du libéralisme politique.

On ne peut qu'être en accord avec Leroux lorsqu'il affirme que notre société a «beaucoup de retard à rattraper sur le plan de la formation à la critique et à la pensée » et en ce qui concerne l'éducation civique (p. 225). Le cours ECR qu'il a contribué à mettre en place viendrait combler en partie ce retard. On applaudit quand il affirme qu'ECR cherche à rétablir « le socratisme démocratique» historiquement dévalorisé au profit d'une "position platonicienne de la vérité et de l'autorité»(p. 226). Nous pensons qu'il s'agit là d'une voie privilégiée pour former à la réflexion critique autonome. C'est d'ailleurs le but du programme: «Proposer une formation à l'éthique, dont le but central est de conduire chaque jeune à une réflexion critique et personnelle sur les grands enjeux moraux de notre temps» (p. $3 \mathrm{I})$.

Mais le fait d'accompagner cette formation éthique d'un enseignement du phénomène religieux est-il pertinent ? Plus précisément, je tenterai d'examiner la réponse de Leroux à cette question essentielle: peut-on concilier l'éducation à la citoyenneté démocratique avec un dialogue non critique de la culture religieuse?

Soyons clairs, il ne s'agit pas du tout de défendre une position hostile aux religions particulières ou au phénomène religieux en général. Comme l'affirme Leroux, la laïcité ne doit surtout pas être une «idéologie antireligieuse militante» (p. 95). Il nous rassure clairement sur ce dont nous étions déjà convaincus et qui aurait pu exacerber cette idéologie: le cours ECR n'est pas une forme d'endoctrinement.

Leroux est aussi très convaincant en ce qui concerne l'importance de la littératie religieuse. L'éducation doit reconnaître la valeur patrimoniale, identitaire et historique des religions, et la connaissance de ce patrimoine participe au développement d'une véritable autonomie citoyenne (p. 40). Mais si l'on peut penser qu'il est primordial de favoriser une riche culture religieuse pour améliorer la connaissance de l'histoire, la compréhension des autres et un meilleur vivre ensemble, en quoi le phénomène religieux doit-il faire partie d'un corpus explicitement dédié au dialogue rationnel et critique?

Un des arguments de Leroux pour légitimer la complémentarité de l'éthique et de la culture religieuse est qu'il faut "considérer comme des 
connaissances de même niveau et de même portée tout ce qui concourt à comprendre les traditions des croyances et des normes au sein de la vie sociale $^{3}$ ». Grâce au pluralisme, les valeurs et les principes qui président à la vie sociale sont d'une diversité remarquable; selon Leroux, la richesse de cette diversité vivifie le dialogue commun démocratique. Le pluralisme n'est toutefois pas un relativisme; au contraire, il est «la recherche interculturelle d'une culture commune» (p. I22) ou, autrement dit, "la recherche active d'une compréhension mutuelle, prenant acte des différences» (p. 234). La diversité des points de vue serait ainsi abordée dans une perspective dialectique de recherche de vérité pour l'élaboration d'une conception morale commune. Le programme trouve donc "son unité dans la poursuite du dialogue démocratique sur tous ces enjeux» (p. 39).

A priori, cette perspective dialectique semble impliquer une dimension critique incontournable et nécessaire pour écarter toute tentation relativiste. Or la dialectique contient un caractère réfutatif, ce qui signifie que, même si tous peuvent prétendre à la vérité, certaines prétentions devront être écartées et conséquemment la diversité devrait s'en trouver immanquablement réduite. Par conséquent, cette conception du dialogue démocratique pourrait entraîner une dévalorisation de certaines conceptions de la vie bonne, qu'elles soient religieuses ou non. On retrouverait ainsi un véritable équilibre entre éthique et culture religieuse «rendu possible notamment par la richesse de la contribution philosophique au dialogue sur les convictions et les croyances et par la primauté de ce dialogue comme compétence dans le programme» (p. I02). Notons que l'auteur, qui s'appuie sur Gadamer et Habermas, définit le dialogue comme la recherche d'une entente qui a pour finalités «l'évolution des consensus sur la détermination des normes de la justice et des conceptions de la vie bonne et la construction critique du savoir moral» (p. I 88). En bref, le programme mettrait dans un équilibre égalitaire l'éthique et la culture religieuse en ce qui concerne leurs prétentions morales et politiques, et cet équilibre serait rendu possible par une véritable «trame critique» (p. 47) au cœur du dialogue démocratique.

Compris ainsi, le programme ne risque-t-il pas de permettre certaines formes d'intolérance ou un manque de respect envers les traditions des croyances qui veulent participer à la fondation d'une culture commune? Pour éviter que le cours légitime une possible intolérance à certaines croyances, le programme accorde un accommodement au discours religieux: il sépare la présentation du phénomène religieux de la réflexion éthique. Cet accommodement se retrouve au cœur même des compétences à atteindre: manifester une compréhension du phénomène religieux et réfléchir sur des questions éthiques. Comme l'auteur l'affirme: "Dans le cas de la culture religieuse, il s'agit d'une compétence de connaissance: à aucun moment l'élève n'est appelé à juger, à évaluer, à critiquer, à comparer ou à

3. Voir le précis de Leroux dans ce numéro. 
discriminer quelque religion ou croyance que ce soit» (p. I75). Contrairement aux conceptions morales inhérentes à la réflexion éthique, "la culture religieuse doit faire l'objet d'un respect absolu» (p. 242). Le concept de respect, que Leroux reprend ici de Kant, implique selon lui de définir une autre forme de dialogue, distinct du débat rationnel éthique qu'il définit ainsi:

Le dialogue sur des questions de culture religieuse interroge les significations et les motifs, mais il s'arrête sur le seuil toujours contingent de la différence: respecter l'autre en tant que différent, c'est d'abord renoncer à mettre en question la croyance au nom d'un universel prescrit par la raison. Il s'agit plutôt de tenter de comprendre, d'approcher les gestes et les croyances en tant que privilèges absolus d'un rapport au monde marqué par l'histoire, par la particularité, et non par une universalisation rationnelle possible (p. 207).

Le dialogue est dans sa nature même, en effet, cet effort de compréhension de l'autre et de soi-même qui nécessite "une réelle sympathie herméneutique, qui se fonde d'abord sur le respect» (p. 36). La raison publique, dans sa recherche d'universalisation rationnelle, ne saurait entreprendre de réduire la diversité religieuse tant que cette diversité est en accord avec les droits fondamentaux intimement liés aux exigences démocratiques.

Après l'introduction du concept de respect absolu, on pourrait croire que Leroux s'oppose à toute remise en question de l'identité religieuse des jeunes. Les jeunes ne seraient donc pas invités à questionner, encore moins à contester les traditions communautaires ou les structures autoritaires liées à la culture religieuse (p. 202):

Car c'est au sein de la famille, et sous la tutelle de l'autorité parentale, que les jeunes développent leurs premières convictions. Il n'est donc pas question de les perturber, encore moins de les mettre en question, mais au contraire d'inviter à les connaître. En toute circonstance, la situation personnelle de chaque jeune sera respectée et l'autorité de la famille reconnue comme ultime (p. I76).

Force est de constater qu'inclure la culture religieuse dans le programme nécessite de modifier fondamentalement le dialogue en y enlevant son essence critique. Leroux nous présente donc, au sein d'un même programme, d'un côté la construction collective critique du savoir moral, et de l'autre un respect absolu de l'autorité. Est-ce conciliable?

Georges Leroux n'est pas sans savoir qu'une compréhension herméneutique comporte toujours une facette critique capitale. La compréhension de l'autre, de son discours, de la réalité ou de l'existence en elle-même est toujours en même temps une mise en question, un examen autant de l'autre que de soi-même. Si le programme valorise la liberté et l'autonomie comme Leroux le prétend, il doit exiger de tous cette attitude herméneutique critique. Même un cours d'histoire moins centré sur le dialogue entraînera un rapport critique comme tout phénomène de compréhension. Sauf que, dans un programme d'histoire où le dialogue collectif n'est pas une compétence explicite, la délibération critique demeurera plus personnelle. En demandant 
explicitement aux étudiants de dialoguer sur la culture religieuse on leur demande de minimiser un des éléments essentiels que par ailleurs dans le même programme on tente de faire émerger: l'esprit critique.

Est-ce à dire que je souhaiterais que la "communauté de recherches » tente de réduire la diversité religieuse par une évaluation rationnelle et critique des différentes conceptions religieuses? Je pense qu'il est important d'établir une distinction entre deux dimensions qui structurent le discours religieux: une dimension symbolique et épistémique ainsi qu'une dimension normative, éthique et politique. Même si ces deux dimensions s'interpénètrent (la dimension normative découle souvent de la dimension symbolique quand elle n'en fait pas partie), elles se distinguent essentiellement par les effets qu'elles peuvent avoir sur autrui.

Une des fonctions principales du discours religieux est de donner un sens à l'existence humaine; expression que l'on peut comprendre au moins de deux façons. Premièrement, dans une dimension symbolique, la religion place l'être humain dans un réseau de significations quant au pourquoi de son existence. On peut croire, par exemple, que Dieu nous a créés, qu'il nous aime, qu'il a un plan pour nous, et que notre existence a un sens qui nous précède. Cette dimension épistémique devrait avoir droit, comme Leroux le souhaite, à un respect absolu, dépourvu de toute évaluation rationnelle. En effet, il est impossible d'évaluer à partir de critères communs la vérité de ces prétentions symboliques. De plus, ces croyances prises en elles-mêmes ont très peu d'effet sur les autres et concernent l'expérience individuelle du croyant.

La deuxième façon de donner un sens à l'existence humaine est éthique et politique parce qu'elle concerne l'action individuelle et collective. Les conceptions religieuses culminent habituellement dans une conception normative qui donne un sens à l'existence parce qu'elle contient des principes qui orientent l'action. Cette deuxième dimension devrait-elle aussi bénéficier d'une absence de critique et d'un respect absolu? Dans l'optique démocratique d'une construction commune d'un vivre ensemble harmonieux, je pense qu'il faut répondre non.

Georges Leroux penserait-il de même? La réponse que l'on peut dégager du livre de Leroux m'apparaît contradictoire. D'une part, il affirme que les positions morales au sein de la culture religieuse doivent être l'objet d'un respect absolu. Par exemple, il affirme que la compassion et la charité ne sont pas des pratiques rationnelles dont on pourrait débattre parce que «nous ne disposons d'aucun argument pour les universaliser en les détachant de leur contexte d'origine» (p. 242). En ce sens, ces positions morales devraient bénéficier d'un respect absolu.

De plus, il soutient que «les convictions religieuses n'intéressent pas en tant que telles le débat public, car elles n'y interviennent pas dans un contexte de rivalité rationnelle ou de collaboration en vue d'un consensus, où il s'agirait pour la société de décider quelles croyances ou quelles pratiques doivent 
être retenues aux fins du bien commun»(p. I69). On peut penser que la compassion, par exemple, impose un respect absolu et ne devrait pas être soumise à l'examen rationnel si elle n'est pas portée sur la place publique et qu'elle sert de principe à des pratiques strictement individuelles. La compassion relèverait alors d'une conviction fondamentale qui fait partie d'une conception particulière de la vie bonne en accord avec nos chartes et les droits fondamentaux. Elle devrait donc aussi profiter d'un respect absolu.

Mais d'autre part, Leroux défend «la nécessité pour chaque jeune de développer une position morale en se fondant sur des principes éthiques acquis au terme d'une réflexion méthodique » (p. I 5 8). Les positions morales peuvent aussi relever de la culture religieuse dans sa dimension normative. S'il faut que les jeunes développent une position morale en se fondant sur des principes éthiques acquis au terme d'une réflexion méthodique et donc critique, cela implique une mise en examen des positions morales religieuses et rendrait inutile, dans cette dimension normative, d'instituer un respect absolu des doctrines religieuses.

Il soutient aussi que des citoyens peuvent être inspirés par des convictions religieuses pour participer au débat public et en conclut que ces positions morales doivent alors être " argumentées, et soumises en tant que telles au processus du consensus par recoupement au sein de la société civile» (p. I70). Il y a donc lieu de s'intéresser aux positions morales religieuses et d'en débattre dans une optique critique, et il est possible de tenter de les justifier rationnellement en les détachant de leur contexte d'origine. C'est aussi ce que Leroux affirme ici en ce qui concerne le dialogue éthique: «Le caractère rationnel de ces discussions n'est certes jamais entièrement imperméable aux croyances particulières [...] le programme les invitera à s'engager dans une démarche rationnelle et dialoguée sur toutes ces questions » (p. 252). N'est-ce pas là mettre la culture religieuse en examen?

Même si, à mon avis, la visée du programme ne peut être la contestation de la tradition et des croyances, l'auteur doit — s'il a à cœur l'autonomie des futurs citoyens - vouloir que les jeunes mettent celles-ci en examen. C'est là le moteur de l'activité philosophique, et on le sait au moins depuis l'enquête de Socrate. C'est aussi le souhait de Leroux, qui reconnaît le danger «d'une contradiction fatale entre le recours à l'autorité et la vie démocratique» (p. 263):

Nous souhaitons que les jeunes comprennent que tout processus conduisant à une décision responsable et exigeant une forme de consensus doit être basé sur le dialogue et non pas d'abord sur l'autorité externe. Telle est la nature de la raison, elle est dialogique et recherche l'autonomie dans son propre exercice. Le rôle de l'autorité ne disparaît pas pour autant, mais celle-ci cesse d'être la source unique de la décision morale (p. 20I).

S'il est vrai que l'enseignant ne peut avoir comme rôle d'inciter directement l'étudiant à questionner ou contester «les structures autoritaires", le pro- 
gramme y incite directement. Peut-on toujours considérer qu'il y a là un respect absolu des croyances religieuses si l'on considère que la plupart reposent sur l'autorité?

Mais le passage le plus marquant, où le respect absolu envers la culture religieuse semble dépassé et dans lequel Leroux invoque Martha Nussbaum est celui où il déclare

qu'il faut d'abord privilégier l'examen critique de soi-même et des traditions qui sont constitutives de notre identité. Toute la formation doit être orientée dans cette direction et invitée à mettre en question le milieu, les habitudes, la culture de classe, la culture nationale, les idéologies du présent, les traditions religieuses, les modèles de performance ${ }^{4}$ (p. 30I).

L'invitation est manifeste, pour les futurs citoyens, à mettre en question l'autorité de leur famille, de leur religion, de leur identité. Comment concilier ce passage avec celui-ci déjà cité: "à aucun moment l'élève n'est appelé à juger, à évaluer, à critiquer, à comparer ou à discriminer quelque religion ou croyance que ce soit» (p. I75) ? À ce stade-ci, doit-on vraiment encore renoncer à remettre la croyance en question, comme nous le prescrit le principe de respect absolu?

Il faut l'affirmer clairement: être éduqué au pluralisme favorise un regard critique sur sa propre conception morale, religieuse ou non, et peut avoir pour conséquence de "déstabiliser la croyance» (p. 7I). Nous devons nous ranger derrière une partie de l'argument des parents de Drummondville qui demandaient l'exemption: le fait d'être mis en présence de la diversité morale ou religieuse met en péril l'éducation dans la foi assurée par les familles. Il ne faut pas tenter de le masquer: tout échange dialectique, c'està-dire éducatif, autant pour l'un que pour l'autre, est toujours une confrontation des identités particulières et transforme un tant soit peu les croyances des individus. Ce qui n'est bien sûr pas un accroc à la liberté de conscience, bien au contraire.

Devant cette apparence de contradiction entre respect absolu et examen critique de la culture religieuse, doit-on vraiment comprendre le respect absolu comme l'absence de possibilité critique? Si l'on revient à la définition la plus substantielle qu'en donne Leroux, il semble que le respect absolu puisse finalement inclure la critique et la remise en question: "Le caractère absolu du respect, au sens kantien de ce terme, désigne donc l'attitude de la conscience engagée envers la dignité des autres et non la soumission cynique au règne de la différence» (p. 243). Le dialogue rationnel et critique ne pourrait-il pas être un moyen privilégié pour s'engager collectivement envers la dignité des autres? Est-il possible d'établir, au sein du phénomène religieux, un dialogue qui soit à la fois critique et respectueux? Il faudrait étendre à la dimension normative de la culture religieuse ce que Leroux avance ici pour l'éthique:

4. Page 30I. D'autres passages vont dans le même sens (p. 4I-236-255). 
Chaque jeune pourra être invité à faire la différence entre les normes qui sont pour lui naturelles et celles qu'il voudrait voir suivies par la collectivité. De la sorte, il sera amené à équilibrer la recherche du bien pour lui et du bien commun collectif, pensé dans une réflexion citoyenne. Mais il n’y a, a priori aucune limite prescriptible à ce travail rationnel, précisément parce que cette réflexion, si elle est menée dans la juste mesure du dialogue, demeurera toujours respectueuse de l'identité de chacun (p. 256).

Pourquoi ce respect critique de l'identité de chacun ne pourrait-il pas s'appliquer aussi à la discussion des positions morales religieuses? Questionner le fondement ou mettre en examen les conceptions morales n'est pas conjoint au mépris et à l'ignorance comme Leroux semble le penser ici: "Le dialogue exige donc que nous entrions en communication, que nous prenions le temps de connaître et d'interroger le sens de nos différences, au lieu de mettre en question le fondement, de mépriser, d'ignorer» (p. 2I 2). Dans le respect absolu des personnes, il est légitime de questionner et de mettre en examen les prétentions éthiques et politiques des croyances pour véritablement interroger le sens de nos différences. Il me semble que c'est là que l'on peut retrouver un véritable respect absolu de la différence et «l'attitude de la conscience engagée envers la dignité des autres et non la soumission cynique au règne de la différence» (p. 243). Et c'est justement ce cadre qui permet de dépasser la compétition stérile qu'identifie Leroux entre les différentes religions et les conceptions séculières pour finalement nous relier vraiment.

L'auteur a raison d'affirmer qu' " une démocratie ne saurait fonctionner si les citoyens n'ont pas les moyens de comprendre les raisons, qu'elles soient religieuses ou morales, croyantes ou athées, soutenant les positions des uns et des autres dans le débat politique et social» (p. 83). Le programme doit promouvoir chez les futurs citoyens une tolérance et une connaissance de la différence. Mais Leroux reconnaît aussi l'autre versant essentiel de la tolérance et de l'ouverture: l'importance d'inculquer une responsabilité à celui qui exprime sa différence, une sorte de devoir de civilité comme John Rawls l'entend, c'est-à-dire la nécessité de justifier ses positions relatives au vivre ensemble par des raisons fondées sur la conception politique de la justice, indépendamment de nos conceptions morales particulières. Cette responsabilité serait-elle plus évidente pour le citoyen si l'on affirmait que toutes les positions morales doivent se soumettre au dialogue critique? Pour éviter toute confusion, ne devrait-on pas reléguer l'étude de ce qui ne peut être critiqué, la dimension symbolique de la culture religieuse, dans un programme différent?

L'un des arguments principaux de Leroux en faveur de la mixité de l'éthique et de la culture religieuse est qu'il faut tenir «compte de l'importance des croyances à l'œuvre dans tant de discussions morales et politiques, qui sont souvent des positions religieuses implicites»(p. 222). L'étudiant doit-il alors adopter la position qu'exige un dialogue de rencontre ou de 
connaissance (p. 2I6), lequel commande le respect absolu de ces positions religieuses et de s'abstenir de les mettre en examen? Qu'en est-il lorsque la position est explicitement religieuse? Je profite de cette disputatio pour demander à Georges Leroux: comment la communauté de recherches en éthique devrait-elle réagir à l'échange qui suit? Que devrait dire l'enseignante ou un autre élève pour concilier le respect absolu et l'attitude critique que nécessite le dialogue éthique?

Enseignante: Pensez-vous qu'il est important pour notre société d'aider les plus démunis?

Élève: Oui

Enseignante: Pourquoi?

Élève: Parce que Dieu affirme qu'il faut s'aimer les uns les autres.

Enseignante: Et pourquoi penses-tu que Dieu affirme cela?

Élève: Parce qu'il connaît la vérité et que je ne peux pas en douter.

Au final, s'il faut accepter un dialogue libre de toute critique dans le respect absolu des croyances en elles-mêmes, il me semble essentiel de le dégager complètement du dialogue philosophique qui, lui, ne peut souffrir d'être non critique. Si Leroux a raison et que chez les étudiants "peu sont indociles ou rebelles, peu sont critiques, très peu cherchent la confrontation des modèles de vie et tentent plutôt de la penser pour eux-mêmes » (p. 306), il ne faut pas leur donner le modèle d'un dialogue non critique en ce qui concerne les positions morales et politiques. Car là où la critique n'est pas possible le dialogue démocratique ne l'est pas non plus.

En remplaçant le cours ECR par un cours de philosophie et d'éducation à la citoyenneté, tout en déplaçant le volet culture religieuse dans un cours d'histoire, nous accomplirions le double programme de Diane L. Moore rapporté par Leroux: la connaissance culturelle des religions et la connaissance philosophique du rôle des religions. La culture religieuse, comme l'affirme Leroux, est a priori relative à l'enseignement de l'histoire, de la civilisation, de la littérature et de la science sociale ${ }^{5}$. Conjointement, un cours de philosophie permettrait une distinction entre les différents types de discours, une réflexion sur la croyance et sur la dimension normative du discours religieux.

J'ai effectué un sondage sans aucune prétention scientifique dans le premier cours de philosophie au collégial que suivent mes étudiants à la session d'automne 2016. Sur I05 étudiants, 70 ont affirmé catégoriquement ne jamais avoir suivi de cours de philosophie auparavant, alors que 2I n'en étaient pas certains. Mais le cours ECR n'est-il pas en grande partie un cours de philosophie? Rien n'est moins sûr: "La mise en question de la croyance par la raison, ou de la raison par la croyance, est l'affaire de la philosophie,

5. Page 8I. Leroux associe la compréhension de la culture religieuse au travail de l'histoire tout au long de l'ouvrage (p. II7-II 8-208-209-2IO-2I3-330). 
elle n'appartient pas à un cours d'éthique et culture religieuse ${ }^{6} »($ p. 22I). Deux des trois compétences de ce cours ne sont-elles pas du ressort de la philosophie? Pour favoriser la légitimité de la philosophie dans l'espace public, il me semble que cela devrait être clair pour les futurs citoyens.

6. Leroux distingue aussi le cours ECR de la philosophie à la page 324 . 